\title{
Reconciling Theory and Experiment in High-resolution Electron Energy-loss Spectroscopy of Plasmon Modes in Individual Nanostructures
}

\author{
A.A. Herzing ${ }^{1}$, X. Zhou ${ }^{2}$, A. Hörl ${ }^{3}$, A. Trügler ${ }^{3}$, U. Hohenester ${ }^{3}$, and T. B. Norris ${ }^{2}$ \\ 1. National Institute of Standards and Technology, 100 Bureau Drive, Gaithersburg, MD 20899, USA \\ 2. Center for Ultrafast Optical Science, University of Michigan, Ann Arbor, Michigan 48109, USA \\ 3. Institut für Physk, Karl-Franzens-Universität Graz, 8010 Graz, Austria
}

Surface plasmon resonances within metal and hybrid nanostructures result from the oscillation of valence electrons in response to an external field [1]. These resonances can strongly dominate the optical response of such structures, and their characteristics are controlled by the composition and geometry of the material. The ability to predictably control these phenomena is enabling a host of new optical and sensing technologies, and further development requires a method for characterizing the local response and understanding the features which effect it. The overall response of an ensemble of such structures can be precisely probed via optical spectroscopy, however, the smallest length scales accessible via these techniques are inadequate for the study of individual resonant modes within a single nanostructure or even those present between structures in an ensemble. In contrast, monochromated electron energy-loss spectroscopy (EELS) in the scanning transmission electron microscope (STEM) offers a route to characterizing these resonance modes in individual nanostructures; combining spatial resolution on the order of a single nanometer and energy resolution on the order of $150 \mathrm{meV} \mathrm{[2-5].}$

Herein, we report our use of spatially-resolved, monochromated STEM-EELS to characterize the plasmonic response in silver nanowires that were $30 \mathrm{~nm}$ and $100 \mathrm{~nm}$ in diameter. Spectral shifts were observed in the plasmon excitation depending on the position of the beam with respect to the tip of the nanowire. In particular, the surface plasmon resonance peak was found to shift to lower energy-loss by $0.3 \mathrm{eV}$ as the beam moved away from the nanowire tip along the direction parallel to the long axis of the wire. In contrast, no such shift was observed when the beam moves away from the side of the wire in a direction perpendicular to the long axis. Additionally, hyperspectral EELS imaging was employed to map the resonant modes along the nanowire surface, within the bulk, and extending into the surrounding vacuum. This technique revealed periodic maxima (Figure 1) in the loss probability near the side surface of the nanowires due to the propagation of lower order modes. These maxima were localized near the surface of the wire but extended a few nanometers into the surrounding vacuum. The characteristics of these modes as well as the surface and bulk modes are readily extracted from the hyperspectral dataset; revealing a great variety in the spectral response of the nanowire as a function of beam position (Figure 2).

These experimental results were interpreted with the aid of three theoretical models: an analytical model for energy-loss in infinite nano-cylinders and nanospheres, numerical simulation of the optical mode intensity, and direct calculation of electron energy-loss using a boundary element method [6]. The models were found to faithfully reproduce many of our experimental observations. The strengths and weaknesses of each will be discussed along with aspects of the experimental work which cause the measured spectra to deviate from theory.

References:

[1] R. H. Ritchie, Phys. Rev. 106 (1957) p. 874. 
[2] M. N'Gom, et al., Nano Lett. 8 (2008) p. 3200.

[3] B. S. Guiton, et al., Nano Lett. 11 (2011) p. 3482.

[4] S. Mazzucco, et al., Nano Lett. 12 (2012) p. 1288.

[5] D. Rossouw, et al., Nano Lett. 11 (2011) p. 1499.

[6] U. Hohenester and A. Trügler, Comp. Phys. Commun. 183 (2012) p. 370.
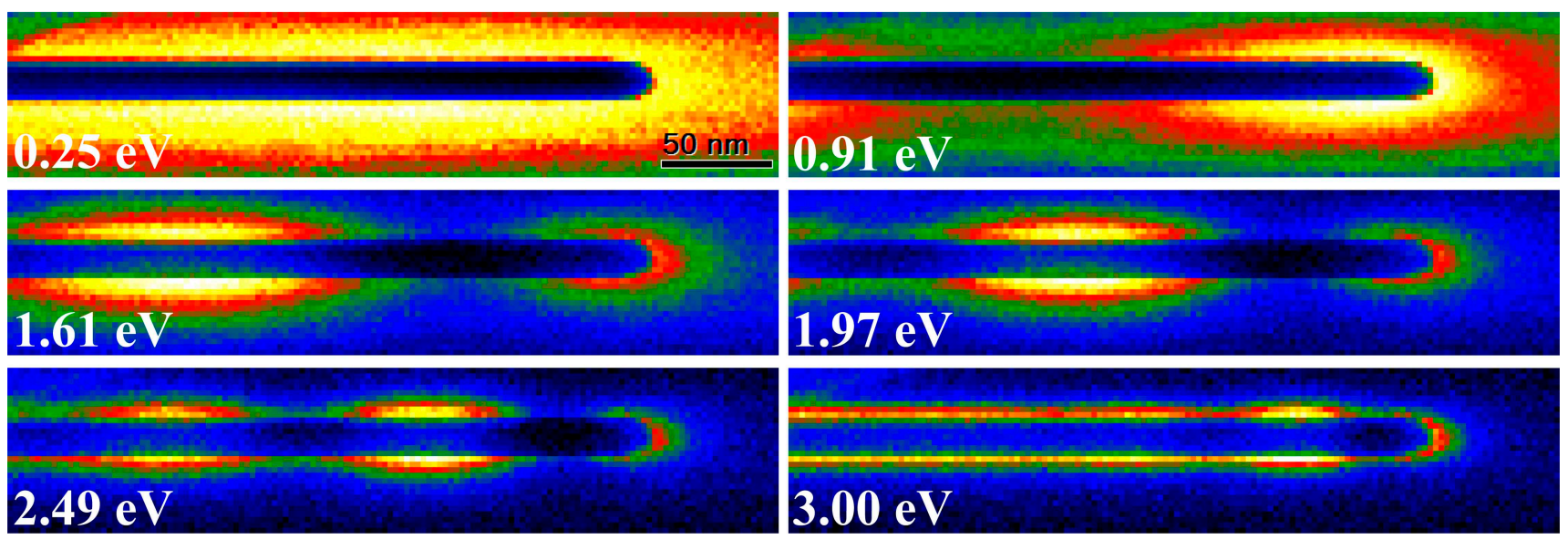

Figure 1 - Intensity maps extracted from a low-loss EELS hyperspectral image data cube showing the spatial distribution of the energy loss response measured from a $35 \mathrm{~nm}$ diameter Ag nanowire.

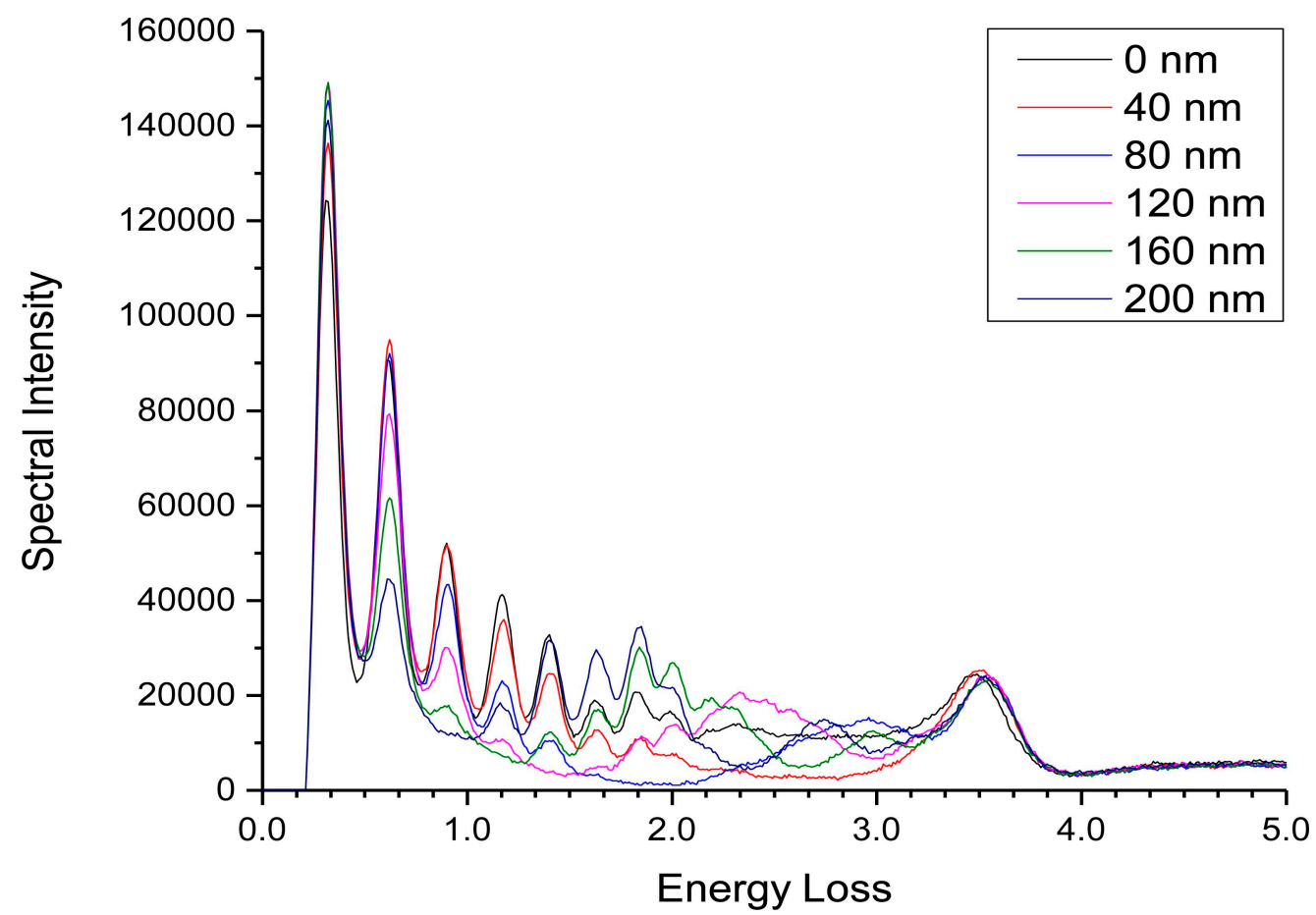

Figure 2 - Spectra extracted from a low-loss EELS hyperspectral image data cube acquired from a $35 \mathrm{~nm}$ diameter Ag nanowire. Spectra were extracted along the edge of the nanowire and shown as a function of distance from the end of the nanowire. 